“ (C) 2018 IEEE. Personal use of this material is permitted. Permission from IEEE must be obtained for all other uses, in any current or future media, including

reprinting/republishing this material for advertising or promotional purposes, creating new collective works, for resale or redistribution to servers or lists, or reuse of any copyrighted component of this work in other works." 


\title{
THE EFFECTIVENESS OF COMPRESSION GARMENTS ON EEG DURING A RUNNING TEST
}

\author{
Thi Nhu Lan Nguyen, Student Member, IEEE, David Eager, Member, IEEE, Hung Tan Nguyen, \\ Senior Member, IEEE
}

\begin{abstract}
The specific purpose of this present paper was to investigate whether the EEG activity has been affected by wearing whole body compression garments during a running test. Ten subjects (men, $n=5$; women, $n=5$; age: $24.11 \pm 4.48$ years; height: $163.56 \pm 7.70 \mathrm{~cm}$; chest: $87.78 \pm 6.92 \mathrm{~cm}$; weight: $58.67 \pm 10.96 \mathrm{~kg}$; BMI: $21.77 \pm 2.63 \mathrm{~kg} . \mathrm{m}^{-2}$ ) completed a running protocol on a treadmill. Each subject participated in two running trials, wearing either a compression garment (CG) or a non-compression garment (NCG) during exercise. Electroencephalogram (EEG) signals were collected during exercise using wearable sensors. The present study revealed a statistically significant difference between CGs and NCGs in alpha, beta and theta power spectral density $(\mathbf{p}<0.05)$. Therefore, the brain activity was influenced by the application of CGs during the running test. This result would also recommends an application of CGs in training as well as in competition.
\end{abstract}

\section{INTRODUCTION}

Fatigue is an effect of physical training and the efficient management of fatigue by the athlete and coach is an imperative task for optimizing performance [1]. Current evidence on development in levels of tiredness has a relationship to fatigue have been demonstrated to the research of declining cognitive attention and slowing motor reaction times, with examples involving a diminished capability to take corrective action and decreased ability to recognize signs of danger [2, 3]. Fatigue has commonly been determined to as reduced alertness and an excessive feeling, which debilitates both willingness and capability to conduct a task [4]. Participants technique in a nonfatigued condition with the best tackling indicated the most significant reduction in tackling technique during fatigued stages. Fatigue concluded in progressive declines in addressing technique [5]. Studies that have reported the relationship between fatigue and sports performance claimed that fatigue is the best deciding factor in the reduction of performance as all viewpoints are inclusive [6].

A number of previous research indicated compression garments (CGs) were associated with fatigue and sports performance. For instance, wearing CGs was relevant to

Thi Nhu Lan Nguyen, David Eager are with the Faculty of Engineering and Information Technology at University of Technology Sydney, New South Waves 2007, Australia, (email: ThiNhuLan.Nguyen@student.uts.edu.au, David.Eager@uts.edu.au).

Hung Nguyen is with the Faculty of Science, Engineering and Technology at Swinburne University of Technology, Victoria 3122, Australia, (email: hungnguyen@swin.edu.au). significantly higher median frequency and lower muscle activation during distance running related to crucial muscles. This result demonstrated that using CGs may enhance muscle function, prevent muscle fatigue and improve running performance [7]. Similarly, compression garments can ease fatigue and, consequently, lead to better performance [8]. CGs may diminish impact accelerations during running, decrease rating of development in the acceleration parameters as fatigue increased [9]. Moreover, CGs may present a low risk for sports injury, reduced fatigue and cause lower muscle activation levels prolonged activity duration. CGs was able to prevent muscle force consumed for stability body's motion which may be wasted on stability on muscle oscillation. That may improve athletic performance [10]. Another study also claimed that wearing CGs during a handball-specific circuit provides benefits on the impairment of the maximal muscle force characteristics and is likely to be worthwhile for handball players involved in activities such as tackles [11]. Driller and Williams recommended the vital role of compression garments in an elite athlete population as a performance and recovery tool [12]. Consistent with that, many research showed CGs might improve performance in sports. For example, a comparison between using lower body CGs and non-CGs demonstrated the improvement of countermovement jump height, the decrease in the rating of perceived exertion post 15 min test and the increase in the level of comfort. Or after submaximal endurance running, lower-limb determined maintenance in muscle power in a group of CGs compared with a group of non-CGs [13]. During a tracking task, upper body CGs showed improvement of performance. It is a useful effect on functional motor control based on reducing in tracking errors [14]. However, the underlying mechanisms by which the impacts of CGs on performance are still lacking.

There was a reduction in muscle activation during a test which was responsible for metabolism and brain oxygenation in a maximal protocol. The decreased cerebral oxygenation might be associated with the activity capacity limiting aspect and the enhancement of central fatigue [15]. The study of the effect of acute effort on EEG claimed that acute exercise could influence on mood variables and brain activity in elderly and young participants [16]. Some conclusions from a systematic review isolated 17 studies claimed that slow wave activity increased in alpha 1 and 2 bands and theta over the entire cortex as person fatigues [17]. The research during a pedaling exercise indicated serotonergic system activation and ventral prefrontal cortex increased alpha band in EEG and induced adverse mood 
development [18]. Similarly, it is clear that EEG excitation was associated with mood which concluded an elemental mechanism of cortical activity after different exercise intensities [19]. Summarizing the main recommendation of the mentioned research, there is a relationship between significant changes in brain wave activity and fatigue condition [17].

To the best of our knowledge, there is no research examining the relationship between using CGs and brain activity based on EEG signals in sports. The aim of this present work is first to examine and measure changes in brain activity that exist as subjects conduct a running test on a treadmill with the application of CGs. We hypothesize that wearing CGs would result in less fatigue.

\section{METHOD}

\section{A. Participants}

Ten healthy participants (men, $\mathrm{n}=5$; women, $\mathrm{n}=5$; age: 24.11 \pm 4.48 years; height: $163.56 \pm 7.70 \mathrm{~cm}$; chest: $87.78 \pm 6.92$ cm; weight: $58.67 \pm 10.96 \mathrm{~kg}$; BMI: $21.77 \pm 2.63 \mathrm{~kg} . \mathrm{m}^{-2}$ ) performed in the running test. All subjects were non-athletes and do not have a smoking history. Exclusion criteria include cardiovascular, respiratory disease, injury or current use of medication. The participants did not consume alcohol and caffeine about 24 hours prior to the experiments. They also were required to have a healthy night sleep.

All volunteers gave a basic medical questionnaire and written consent documents before the test. The approval number is UTS HREC REF NO. ETH16-0696, which given by University of Technology Sydney Human Ethic Committee. The detailed characteristics of participants (five men and five women) are shown in Table 1.

\section{B. Study protocol}

The protocol was designed for each participant visited the laboratory two times throughout the trials period which was conducted by at least two separate days. CGs and NCGs were randomized during the two running tests. The normal laboratory temperature was set at a range of $20-22^{\circ} \mathrm{C}$.

The CGs used in the current experiment was SportSkins Classic whole body compression garments (WBCGs), from Skins, Campbelltown, NSW, Australia. The whole body CGs included long-leg pants (from waist to ankle) and a longsleeved top (from neck and wrist to waist) which comprised of $24 \%$ Roica Spandex and $76 \%$ Nylon and Meryl Microfiber. Each CGs was fitted with size-correct garments according to the manufacturer's guideline (https://www.skins.net/au/size-guide/), based on subjects' body mass and stature. During the experiments, NCGs was a $\mathrm{T}$-shirt and short with $0 \%$ level of pressure. In both tests, participants were required to wear the same socks and shoes. The kind of comparison garments is shown in Figure 1.

Subjects participated in two running tests with CGs and without CGs on two different days. A rest of about 10 minutes before the tests was required. EEG electrodes were attached to the O1 position (Figure 1). Data of EEG-rest were collected after this step.
Participants performed a 10-minute running at $6 \mathrm{~km} / \mathrm{h}$ on $0 \%$ grade of a treadmill. The speed then increased by $1 \mathrm{~km} / \mathrm{h}$ every 2-minute running. Participants required stopping about 20 seconds to collect data after completing each velocity. The process continued until a speed of $11 \mathrm{~km} / \mathrm{h}$ was reached. EEG signals were obtained during the exercise using a Flexcomp Infiniti Monitor of Thought Technology Ltd, Canada. Impedance was checked before and after the test following Figure 2.

TABLE I. SUBJECTS CHARACTERISTICS

\begin{tabular}{|l|l|l|}
\hline \multirow{2}{*}{\multicolumn{1}{|c|}{ Parameters }} & \multicolumn{2}{|c|}{ Subjects (n=10) } \\
\cline { 2 - 3 } & $\begin{array}{l}\text { Men (n=5) } \\
\text { Mean } \pm \text { std }\end{array}$ & $\begin{array}{l}\text { Women (n=5) } \\
\text { Mean } \pm \text { std }\end{array}$ \\
\hline Age (year) & $25.50 \pm 5.26$ & $23.00 \pm 4.00$ \\
\hline Height $(\mathrm{cm})$ & $170.25 \pm 4.50$ & $158.20 \pm 4.76$ \\
\hline Chest $(\mathrm{cm})$ & $93.75 \pm 1.85$ & $83.00 \pm 5.39$ \\
\hline Weight $(\mathrm{kg})$ & $67.75 \pm 5.74$ & $51.40 \pm 8.20$ \\
\hline Body mass index (kg.m-2) & $23.43 \pm 2.47$ & $20.46 \pm 2.09$ \\
\hline
\end{tabular}

Figure 1. Subject wear compression garments and non-compression garments

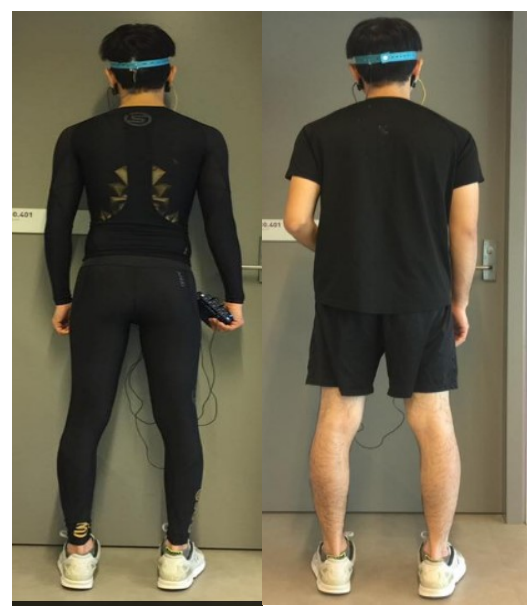

Figure 2. Impedance-check before and after the test.
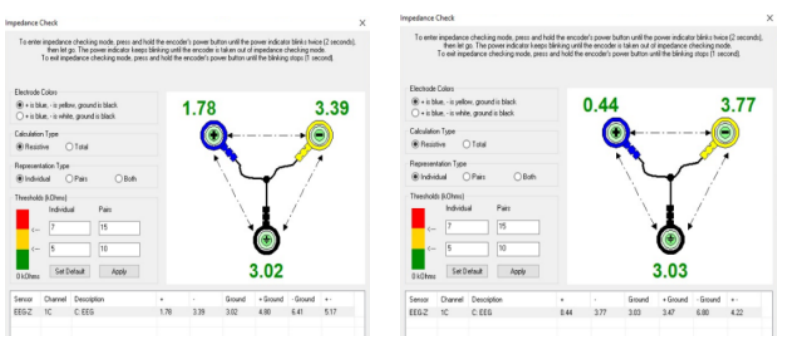

\section{Data analysis}

The mean value and standard deviation were computed for all descriptive and physiological measures. All analyses were calculated with Matlab version 2016b.

EEG data during the walking and running were chosen during 20 seconds each completed velocity when participants stopped. Parameters were compared between two grament conditions based on paired t-tests. Statistical significance is reported when the p-value is lower than 0.05 . 


\section{RESULTS}

During exercise, a significant increase in power spectral density across both $\mathrm{CG}$ and NCG groups was reported $(p<0.05)$. However, $C G$ group experienced substantially lower alpha power spectral density compared with NCG group at $7 \mathrm{~km} / \mathrm{h}$ (CGs: 20.95 , NCGs: 31.85 , $\mathrm{p}=0.0081$ ), at 8 $\mathrm{km} / \mathrm{h}$ (CGs: 29.79, NCGs: 41.44, $\mathrm{p}=0.0016$ ), at $9 \mathrm{~km} / \mathrm{h}$ (CGs: 34.80, NCGs: 47.55, p=0.0062), at $10 \mathrm{~km} / \mathrm{h}$ (CGs: 39.74, NCGs: 53.01, p=0.0131) and $11 \mathrm{~km} / \mathrm{h}$ (CGs: 41.41, NCGs: $55.75, \mathrm{p}=0.0071$ ). The results are shown in Figure 3.

Similarly, high alpha and low alpha also indicated the significant difference from $7 \mathrm{~km} / \mathrm{h}$ to $11 \mathrm{~km} / \mathrm{h}$ between two garment groups. High alpha showed significant difference between CGs and NCGs at $7 \mathrm{~km} / \mathrm{h}$ (CGs: 14.56, NCGs: 21.44, $\mathrm{p}=0.0101$ ), at $8 \mathrm{~km} / \mathrm{h}$ (CGs: 20.29, NCGs: 27.17, $\mathrm{p}=0.0007)$, at $9 \mathrm{~km} / \mathrm{h}$ (CGs: 23.82, NCGs: 30.63, $\mathrm{p}=0.0071)$, at $10 \mathrm{~km} / \mathrm{h}$ (CGs: 27.00 , NCGs: $33.76, \mathrm{p}=0.0244)$, at $11 \mathrm{~km} / \mathrm{h}$ (CGs: 28.08, NCGs: 35.13, $\mathrm{p}=0.014)$. The results are shown in Figure 4. Consistent with that, low alpha illustrated significantly lower values in CGs when compared to NCGs at 7 km/h (CGs: 15.43, NCGs: 24.37, p=0.0084), 8 km/h (CGs: 22.85, NCGs: 32.54, $\mathrm{p}=0.0044$ ), at $9 \mathrm{~km} / \mathrm{h}$ (CGs: 26.71, NCGs: $37.95, \mathrm{p}=0.0115$ ), at $10 \mathrm{~km} / \mathrm{h}$ (CGs: 30.72, NCGs: 42.73, $\mathrm{p}=0.0192)$ and at $11 \mathrm{~km} / \mathrm{h}$ (CGs: 32.04, NCGs: 45.25, $\mathrm{p}=0.0120$ ). The results are shown in Figure 5.

Additionally, beta power spectral density was found to have significantly increased in both CGs and NCGs group during exercise $(\mathrm{p}<0.05)$. Beta parameters demonstrated the significant difference between CGs and NCGs at $7 \mathrm{~km} / \mathrm{h}$ (CGs: 14.64, NCGs: 18.78, $\mathrm{p}=0.0291$ ), at $8 \mathrm{~km} / \mathrm{h}$ (CGs: 17.43, NCGs: 22.00, $\mathrm{p}=0.0147$ ), at $9 \mathrm{~km} / \mathrm{h}$ (CGs: 19.51, NCGs: 23.75, p=0.037), at $10 \mathrm{~km} / \mathrm{h}$ (CGs: 21.33, NCGs: 26.04, $\mathrm{p}=0.0282)$ and at $11 \mathrm{~km} / \mathrm{h}$ (CGs: 22.11, NCGs: 27.18, $\mathrm{p}=0.024)$. The results are presented in Figure 6 .

Similar result was forthcoming for theta power spectral density. Substantial increases also occurred in theta from velocity of $6 \mathrm{~km} / \mathrm{h}$ to the end of exercise at $11 \mathrm{~km} / \mathrm{h}$ in both groups of garments $(\mathrm{p}<0.05)$. The significant difference between CGs and NCGs revealed at $6 \mathrm{~km} / \mathrm{h}$ (CGs: 24.25, NCGs: 44.11, p=0.007), $7 \mathrm{~km} / \mathrm{h}$ (CGs: 28.23, NCGs: 56.76, $\mathrm{p}=0.0025), 8 \mathrm{~km} / \mathrm{h}$ (CGs: 46.21, NCGs: 72.29, $\mathrm{p}=0.0225), 9$ $\mathrm{km} / \mathrm{h}$ (CGs: 51.27 , NCGs: $81.48, \mathrm{p}=0.0181), 10 \mathrm{~km} / \mathrm{h}$ (CGs: 56.90, NCGs: $90.79, \mathrm{p}=0.0146$ ) and $11 \mathrm{~km} / \mathrm{h}$ (CGs: 59.28, NCGs: 94.75, $\mathrm{p}=0.0089)$. The results are presented in Figure 7.

Figure 3. Alpha-power spectral density $\left({ }^{* *} \mathrm{p}<0.01,{ }^{*} \mathrm{p}<0.05\right)$

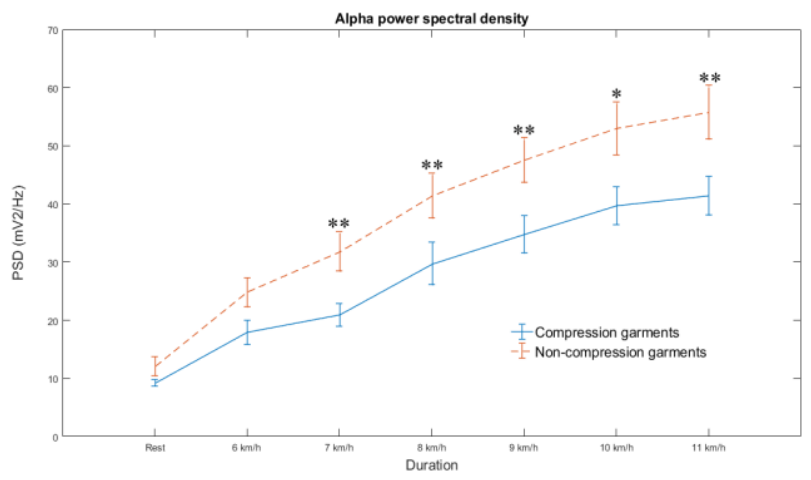

Figure 4. High alpha $\left({ }^{* * *} \mathrm{p}<0.001,{ }^{* *} \mathrm{p}<0.01,{ }^{*} \mathrm{p}<0.05\right)$

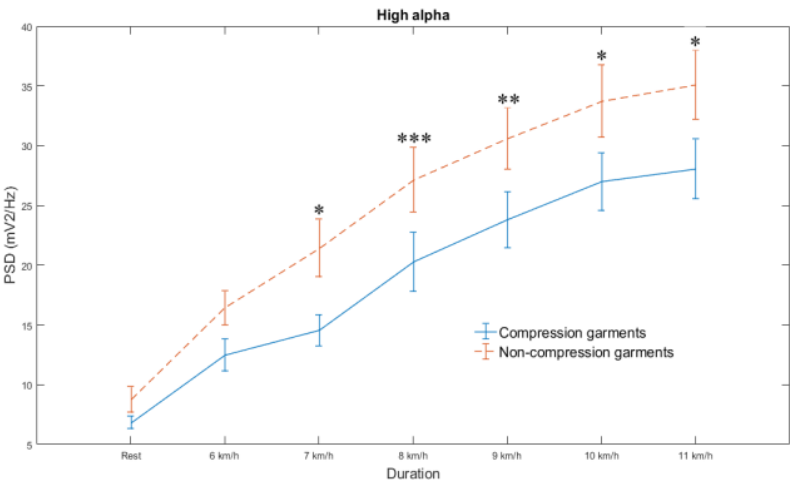

Figure 5. Low alpha $\left({ }^{* *} \mathrm{p}<0.01,{ }^{*} \mathrm{p}<0.05\right)$

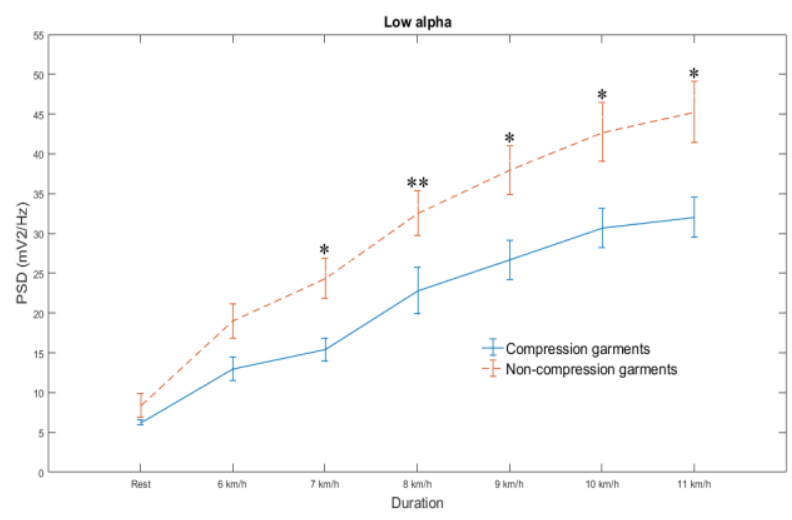

Figure 6. Beta power spectral density $(* \mathrm{p}<0.05)$

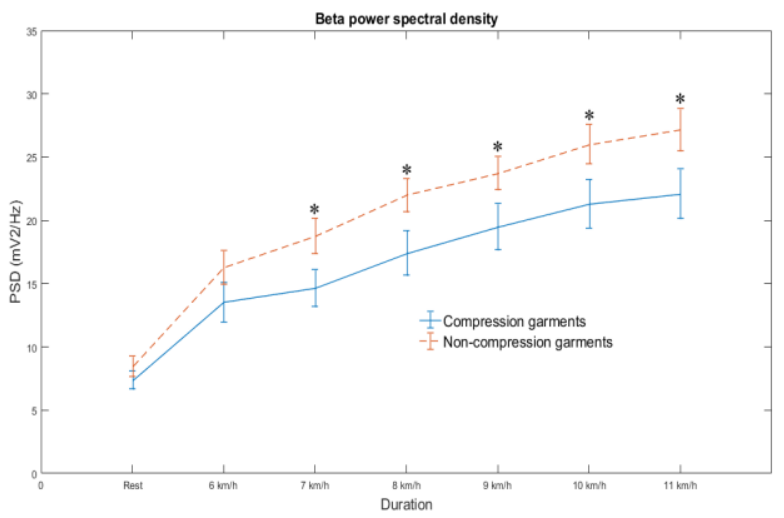

Figure 7. Theta-power spectral density $(* * p<0.01, * \mathrm{p}<0.05)$

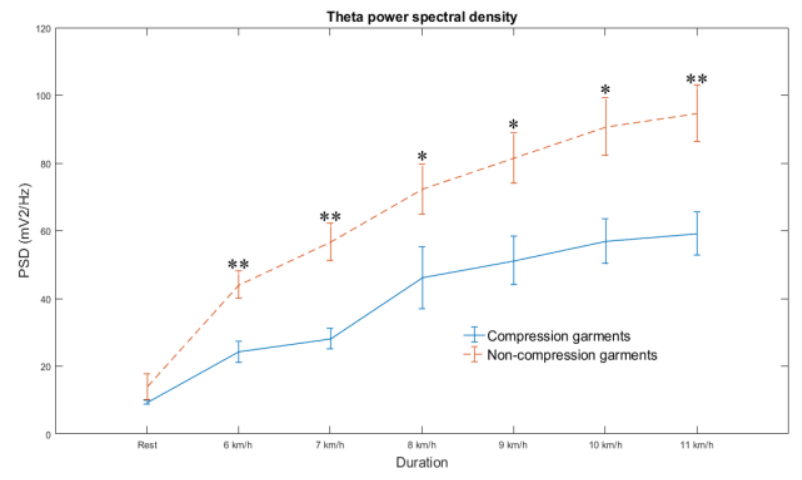




\section{Discussion AND CONCLUSION}

This present research is the first to simultaneously assess brain activity with EEG signals to suggest the influence of CGs during the running exercise. It examined EEG wave power spectral density significantly improved during the protocol in both groups of garments. Following a paradigm put forward in previous work suggesting that beta power indicated a significant increase at central (C4) and frontal (Fp1, F3, and F4) areas, which associated with the improvement of cortical activation [20]. Moreover, the finding is consistent with research of EEG in an aerobic exercise which reported a significant enhancement in an alpha wave with low-frequency range and theta wave related to acute activity with low intensity [21]. Additionally, CGs indicated the significantly lower values in all parameters including alpha, beta and theta compared with NCGs from 7 $\mathrm{km} / \mathrm{h}$ to $11 \mathrm{~km} / \mathrm{h}(\mathrm{p}<0.05)$. These evidence demonstrated CGs effects on brain activity during the running test.

The previous study from our research team evaluated the relationship between fatigue with the changes of regional brain wave activity. The results demonstrated that alpha 1, alpha 2 bands and theta increased over the entire cortex in fatigued participants [17]. Similarly, alteration in cortical activity estimated throughout a high-intensity cycling exercise identified a significant development in EEG power during the test as fatigue increased [22]. The findings of the current study concluded that alpha power spectral density, high alpha, low alpha, theta power spectral density and beta power spectral density indicated significantly higher value in NCGs group compared with CGs group over the occipital lobes.

Although limitations exist, the results support the hypotheses that wearing CGs would reduce fatigue and improve performance. This observation would also suggest an application of CGs in training as well as in competition.

\section{ACKNOWLEDGMENT}

The appreciation of researchers is given to participants The University of Technology, Sydney students for their attendance and Mr. Raymond Clout - the UTS lab managers for his technical support.

\section{REFERENCES}

[1] P. J. Robson-Ansley, M. Gleeson, and L. Ansley, "Fatigue management in the preparation of Olympic athletes," Journal of sports sciences, vol. 27, pp. 1409-1420, 2009.

[2] K. Taylor, D. Chapman, J. Cronin, M. J. Newton, and N. Gill, "Fatigue monitoring in high-performance sport: a survey of current trends," J Aust Strength Cond, vol. 20, pp. 12-23, 2012.

[3] Z. Wang, R. Zheng, T. Kaizuka, K. Shimono, and K. Nakano, "The Effect of a Haptic Guidance Steering System on FatigueRelated Driver Behavior," IEEE Transactions on HumanMachine Systems, vol. 47, pp. 741-748, 2017.

[4] D. Dreiling, "Fatigue and exercise," Home health care management \& practice, vol. 22, pp. 34-39, 2009.

[5] T. J. Gabbett, "Influence of fatigue on tackling technique in rugby league players," The Journal of Strength \& Conditioning Research, vol. 22, pp. 625-632, 2008.
[6] A. J. Knicker, I. Renshaw, A. R. Oldham, and S. P. Cairns, "Interactive processes link the multiple symptoms of fatigue in sport competition," Sports Medicine, vol. 41, pp. 307-328, 2011.

[7] W.-C. Hsu, L.-W. Tseng, F.-C. Chen, L.-C. Wang, W.-W. Yang, Y.-J. Lin, et al., "Effects of compression garments on surface EMG and physiological responses during and after distance running," Journal of Sport and Health Science, 2017.

[8] G. Chin-Yi, C.-H. Chen, Y.-M. Sun, and L.-1. Wang, "The Effects Of Compression Garments On Sports Performance And Fatigue," in ISBS-Conference Proceedings Archive, 2016.

[9] A. Lucas-Cuevas, J. Priego-Quesada, I. Aparicio, J. Giménez, S. Llana-Belloch, and P. Pérez-Soriano, "Effect of 3 Weeks Use of Compression Garments on Stride and Impact Shock during a Fatiguing Run," International journal of sports medicine, vol. 36, pp. 826-831, 2015.

[10] P. Wang, L. Li, M.-d. Yan, and F. Ru, "Effects of Compression Garments on Lower Limb Muscle Activation via Electromyography Analysis during Running," 2014.

[11] G. Ravier, R. Bouzigon, S. Beliard, N. Tordi, and F. Grappe, "Benefits Of Compression Garments Worn During HandballSpecific Circuit On Short-Term Fatigue In Professional Players," Journal of strength and conditioning research/National Strength \& Conditioning Association, 2016.

[12] M. W. Driller and N. B. Williams, "The use of compression garments in elite australian athletes: a survey," Journal of Athletic Enhancement, vol. 2016, 2016.

[13] S. Rugg and E. Sternlicht, "The Effect of Graduated Compression Tights, Compared With Running Shorts, on Counter Movement Jump Performance Before and After Submaximal Running," Journal of Strength \& Conditioning Research, vol. 27, pp. 1067-1073, 2013.

[14] A. J. Pearce, D. J. Kidgell, L. A. Grikepelis, and J. S. Carlson, "Wearing a sports compression garment on the performance of visuomotor tracking following eccentric exercise: A pilot study," Journal of Science and Medicine in Sport, vol. 12, pp. 500-502, $7 / / 2009$.

[15] P. Rasmussen, J. Nielsen, M. Overgaard, R. Krogh-Madsen, A Gjedde, N. Secher, et al., "Reduced muscle activation during exercise related to brain oxygenation and metabolism in humans," The Journal of physiology, vol. 588, pp. 1985-1995, 2010.

[16] H. Moraes, A. Deslandes, H. Silveira, P. Ribeiro, M. Cagy, R. Piedade, et al., "The effect of acute effort on EEG in healthy young and elderly subjects," European journal of applied physiology, vol. 111, pp. 67-75, 2011.

[17] A. Craig, Y. Tran, N. Wijesuriya, and H. Nguyen, "Regional brain wave activity changes associated with fatigue," Psychophysiology, vol. 49, pp. 574-582, 2012.

[18] M. Fumoto, T. Oshima, K. Kamiya, H. Kikuchi, Y. Seki, Y. Nakatani, et al., "Ventral prefrontal cortex and serotonergic system activation during pedaling exercise induces negative mood improvement and increased alpha band in EEG," Behavioural brain research, vol. 213, pp. 1-9, 2010.

[19] S. Schneider, C. D. Askew, J. Diehl, A. Mierau, J. Kleinert, T. Abel, et al., "EEG activity and mood in health orientated runners after different exercise intensities," Physiology \& behavior, vol. 96, pp. 709-716, 2009.

H. Moraes, C. Ferreira, A. Deslandes, M. Cagy, F. Pompeu, P. Ribeiro, et al., "Beta and alpha electroencephalographic activity changes after acute exercise," Arquivos de neuro-psiquiatria, vol. 65, pp. 637-641, 2007.

[21] S. Nishifuji, "EEG recovery enhanced by acute aerobic exercise after performing mental task with listening to unpleasant sound," in Engineering in Medicine and Biology Society, EMBC, 2011 Annual International Conference of the IEEE, 2011, pp. 38373840.

[22] H. Enders, F. Cortese, C. Maurer, J. Baltich, A. B. Protzner, and B. M. Nigg, "Changes in cortical activity measured with EEG during a high-intensity cycling exercise," Journal of neurophysiology, vol. 115, pp. 379-388, 2016. 